\title{
Ultrasonic Assisted Photolytic Degradation of Reactive Black 5 [RB5] Simulated Wastewater
}

\author{
Collin G. Joseph *,1 \\ Yun Hin Taufiq-Yap ${ }^{2}$ \\ Vigneswar Krishnan ${ }^{1}$ \\ 1 Sonophotochemistry Research Group, Faculty of Science and Natural Resources, Universiti \\ Malaysia Sabah, 88400 Kota Kinabalu, Sabah \\ ${ }^{2}$ Centre of Excellence for Catalysis Science and Technology, Faculty of Science, Universiti Putra \\ Malaysia, 43400 UPM Serdang \\ *e-mail: collin@ums.edu.my
}

The present study is based on a hybrid advanced oxidation process (AOP) of sonophotolysis system, of which sonication $(35 \mathrm{kHz})$ and photolysis UV-C (254nm) were applied simultaneously to effectively degrade a selected recalcitrant dye-based pollutant, Reactive Black 5 (RB5). The influence of the solution $\mathrm{pH}$ and concentration were manipulated throughout this study to investigate the sonophotodegradation kinetics and synergistic effects on the RB5 degradation. Increasing the solution concentration resulted in lowered degradation rate due to the inner filter effect by the dye molecules and reduced the generation of hydroxyl radicals. The results confirmed that the sonophotolysis rate was better at basic medium (67.7\%) in comparison to acidic medium (46.9\%) due to the ionization of RB5. Synergistic effects were analyzed based on the first order kinetic rate model. It was found that the synergistic effect was observed for all the experiments conducted which resulted from an increase in the $(\cdot \mathrm{OH})$ radicals due to the photolysis of $\mathrm{H}_{2} \mathrm{O}_{2}$ formed by the sonolysis process. This hybrid system, sonophotolysis system, was able to degrade RB5 into intermediates with a total reaction time of $1 \mathrm{~h}$.

Keywords: Sonolysis; Photolysis; Sonophotolysis; RB5

\section{INTRODUCTION}

Water from the textile industry has been of concern to researchers because they are characterized by the prominent colour and COD content with varying $\mathrm{pH}$ from 2 to 12 , resulting in polluted water bodies like lakes and rivers becoming toxic to aquatic life (Ramirez et al., 2013). According to Rodríguez et al., (2009) the colour and structural integrity of dyes in textiles effluents are hardy and degrade very little in the environment due to their design to persist under oxidizing and reducing conditions or washing. Dyes are resistant to fading by exposure to sunlight, water and many chemicals such as detergents due to their complex structure and synthetic origin. The structural varieties of dyes are acidic, basic, disperse, azo, diazo, anthraquinone based and metal complex dye. The municipal sewerage systems do not affect the decolourisation of textile dye when it is treated aerobically (Robinson et 
al., 2001).

Current conventional methods such as membrane technology, filtration, coagulation, and flocculation are used to treat the dyehouse effluent. Most of the conventional methods are ineffective to sufficiently address the treatment due to the complexity of the composition of dye house effluent (Mohan \& Balasubramanian, 2006). The production of the huge amount of chemical sludge and the ineffectiveness in decolourising of some soluble dyes by these treatment methods are well documented (Mezohegyi et al., 2012). The limitations of conventional chemical oxidation techniques can be overcome by treating this type of wastewater with advanced oxidation process (AOP) methods (Alaton et al., 2002). AOPs, which include treatment with sonication, ozone, $\mathrm{H}_{2} \mathrm{O}_{2}$, Fenton's reagent or UV radiation are the potential alternatives for the traditional decolourisation methods for dye contaminated wastewater. AOPs are a combination of non-biological technologies which are involved in the degradation of toxic pollutants such as biologically recalcitrant compounds in textile wastewater (Wang et al., 2003). AOPs generally rely on the generation of reactive hydroxyl radical with a redox potential of $2.8 \mathrm{~V}$. Hydroxyl radical rapidly reacts with the organic compound, either by addition to a double bond or by abstraction of a hydrogen atom from an aliphatic organic molecule. The organic radicals formed will then react with oxygen to initiate a series of oxidation reactions ultimately leading to mineralized products of $\mathrm{CO}_{2}$ and $\mathrm{H}_{2} \mathrm{O}$ (Catalkaya et al., 2009).

Sono-hybrid processes have been proposed and investigated by many researchers as shown in the literature, such as the sono-persulfate process, sonoFenton process, sono-electrochemical process, sono-photoferrioxalate process and sonophotolytic process. Among these hybrid processes, the sonophotolytic process shows more promise for practical application due to its avoidance of chemical addition. Sonophotolysis involves the practical application of combining ultrasonic sound waves (US) $(20-1000 \mathrm{kHz})$ and ultraviolet radiation (UV) to enhance a chemical reaction by the formation of free radicals in aqueous systems. Ultrasonic sound waves are transmitted through the aqueous solution to generate an "acoustic cavitation" (Duran et al., 2013). During this combination, the synergistic effect of the US waves and the UV irradiation has resulted in improved degradation efficiencies (Monteagudo et al., 2014; Bahena et al., 2008; Joseph et al., 2015; Xu et al., 2013).

The aim of the present work is to investigate the effect of light irradiation wavelength, the influence of $\mathrm{pH}$ and solution concentration on the sonophotolytic decomposition of an azo dye, called Reactive Black 5(RB5). The synergistic effect of solution concentration and solution $\mathrm{pH}$ were studied and presented.

\section{MATERIALS AND METHOD}

\section{Materials}

Azo dye C.I. Reactive black (RB5) with $55 \%$ purity was obtained from SigmaAldrich. $\mathrm{HCl}$ and $\mathrm{NaOH}$, which were used for $\mathrm{pH}$ adjustments, were purchased from 


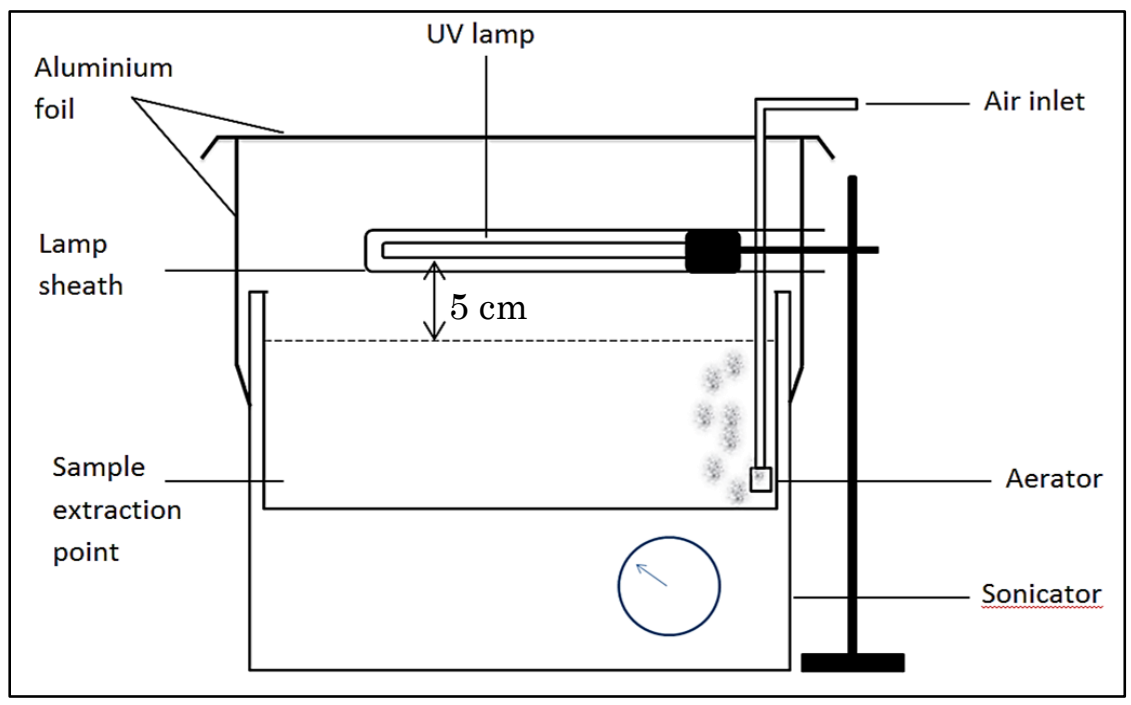

Fig. 1: Schematic diagram of sonophotoreactor

Fischer Scientific (37\%) and Sigma-Aldrich $(\geq 98 \%)$, respectively. These chemicals were used without further modification.

\section{Ultrasonic Bath}

Ultrasonic bath (Bandelin sonorex RK31) with the dimensions of $190 \mathrm{~mm}$ in length, $85 \mathrm{~mm}$ in width and $60 \mathrm{~mm}$ in height was employed in this experiment. It has an operating frequency of $35 \mathrm{kHz}$ and rated power output of $120 \mathrm{~W}$. Both frequency and power output were kept constant for all experiments. The bath is made from stainless steel with large surface area for the PZT transducers to produce a uniform ultrasonic intensity throughout the entire oscillating tank. The cavitational yield $(\mathrm{mg} / \mathrm{J})$ defined as net degradation $(\mathrm{mg} / \mathrm{L})$ per unit of power density $(\mathrm{J} / \mathrm{L})$ was calculated for all experiments involving the use of ultrasound. The formula used in the calculations of the cavitational yield is as follows (Barik et al., 2016):

Cavitational yield $=\frac{\text { Degradation in } \mathrm{mg} / \mathrm{L}}{\text { Power density in } \mathrm{J} / \mathrm{L}}$

\section{Experimental Procedure}

The sonophotoreactor was a batch type reactor system as shown (in Fig. 1). The lamp experimented in this study were $9 \mathrm{~W}$ UV-A (365nm) lamp from Sylvania, 9W UVB (311nm) lamp from Philips, 9W (Pro UV, 254nm) UV-C lamp also from Philips (Model: TUVPL-S9W/2P1CT) and 9W Solar (maximum peak at $610 \mathrm{~nm}$ ) lamp from Coralife. The light intensity for the lamps according to the manufacturer's specification were listed as $10 \mathrm{~mW} / \mathrm{cm}^{2}$ while all the lamps had a total radiating surface area of $91.7 \mathrm{~cm}^{2}$. The lamp was mounted inside a quartz lamp sheath which was placed horizontally on top of sonicator at a distance of $5 \mathrm{~cm}$ from the reacting solution. The experiments were carried out by varying RB5 solution from $5 \mathrm{ppm}$ to $25 \mathrm{ppm}$.

The solution $\mathrm{pH}$ was adjusted using 0.10 $\mathrm{M} \mathrm{HCl}$ for acidic $\mathrm{pH}$ or $0.10 \mathrm{M} \mathrm{NaOH}$ for alkaline pH (Zhang et al., 2013). Samples in each experiment were extracted on a 10 minute interval throughout the 1 hour experiment. The samples were extracted using a syringe and transferred into vials. 
The samples were analysed by UV-vis spectroscopy (Lambda 25 UV-vis Spectrophotometer). All experiments were done in triplicates with their average values taken and plotted into graphs. Photo. 1 shows the top view of batch sonophotoreactor without the aluminium cover. The concentration of RB5 was determined at the peak absorption at $595 \mathrm{~nm}$ from a calibration curve determined using 5, 10, 15, 20 and 25ppm RB5. The entire sonophotoreactor was covered using a black polyethylene bags to prevent interference from ambient light and to prevent exposure to UV radiation.

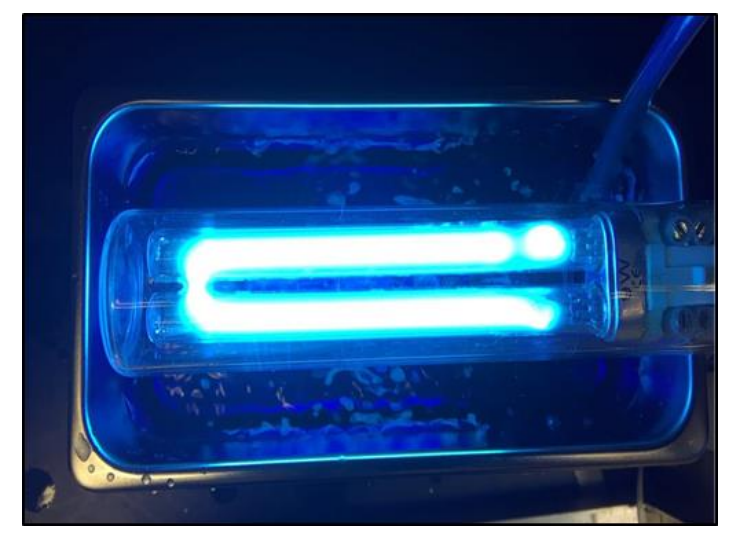

Photo 1: Top view of sonophotoreactor (without aluminium foil cover)

\section{Reaction Kinetics}

The degradation of RB5 over a time period $t$ was determined using (2):

$$
D_{g}=\frac{C_{0}-C_{t}}{C_{0}} \times 100 \%
$$

where $\% D_{g}$ is the extent of degradation; $C_{0}$ is the initial solution concentration; $C_{t}$ is the concentration of sample at time, $t$ the time in minutes. In this study, the first-order rate constant was determined from the linear fitting of a plot of $\ln \left(C_{t} / C_{0}\right)$, against time $(t)$ according to (3).

$$
\ln \frac{C_{t}}{C_{0}}=-k t
$$

where $C_{0}$ is the initial solution concentration; $C_{t}$ is the concentration of the sample after time, $t ; k$ is the rate constant and $t$ is the time.

\section{Calculation of Synergy}

The mechanistic interactions among the individual AOPs were determined on the basis of synergy (Chakma et al., 2016). The synergism between sonolysis, photolysis, and hybrid system, sonophotolysis was calculated using the first-order rate constants as follows (4) (Joseph et al., 2015; Duran et al., 2013).

Synergy, $(S)$

$=\frac{k_{\text {sonophotolysis }}-\left(k_{\text {sonolysis }}+k_{\text {photolysis }}\right)}{k_{\text {sonophotolysis }}}$

\section{RESULTS AND DISCUSSION}

Previous studies have shown that sonolysis or photolysis conducted individually was not effective in degrading RB5 (Zhou et al., 2009). Fig. 2(a) shows the effect of solution concentration on the RB5 sonophotolytic degradation constants at a frequency of $35 \mathrm{kHz}$. The degradation rate increased with concentration decreased for all three systems investigated. These figures show that the concentration of aqueous solution of azo-dye has a significant effect on the degradation rates. It can be concluded that the degradation of lower concentration RB5 took a shorter time than that of higher concentration of the RB5 solution. It is known that the production of ${ }^{\bullet} \mathrm{OH}$ remains constant for a given sonication power and time (Zhou et 


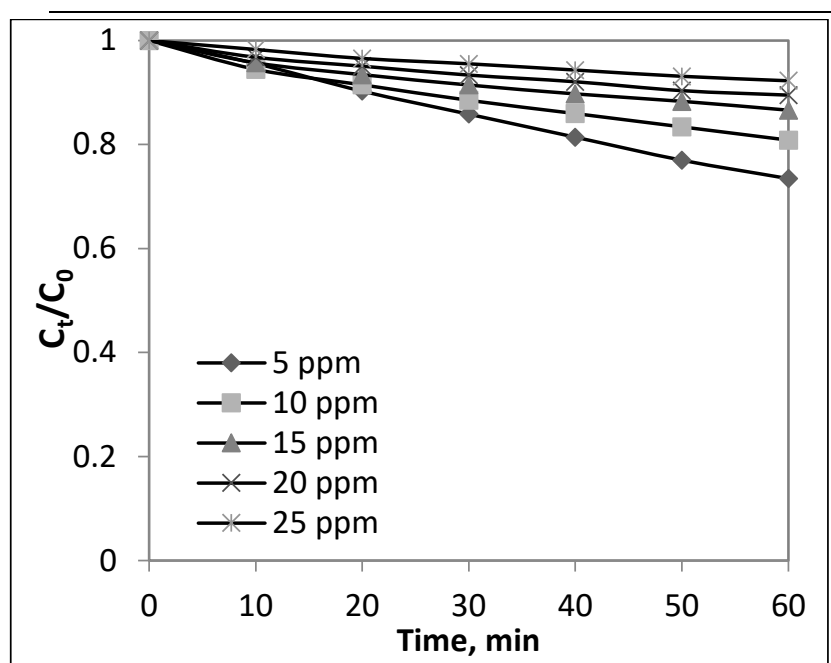

(a)

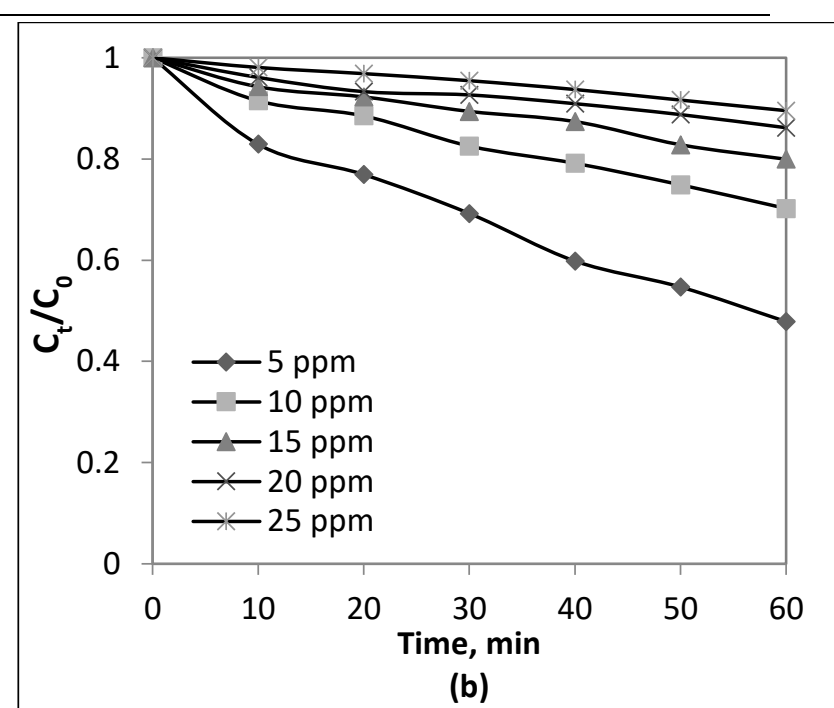

(b)

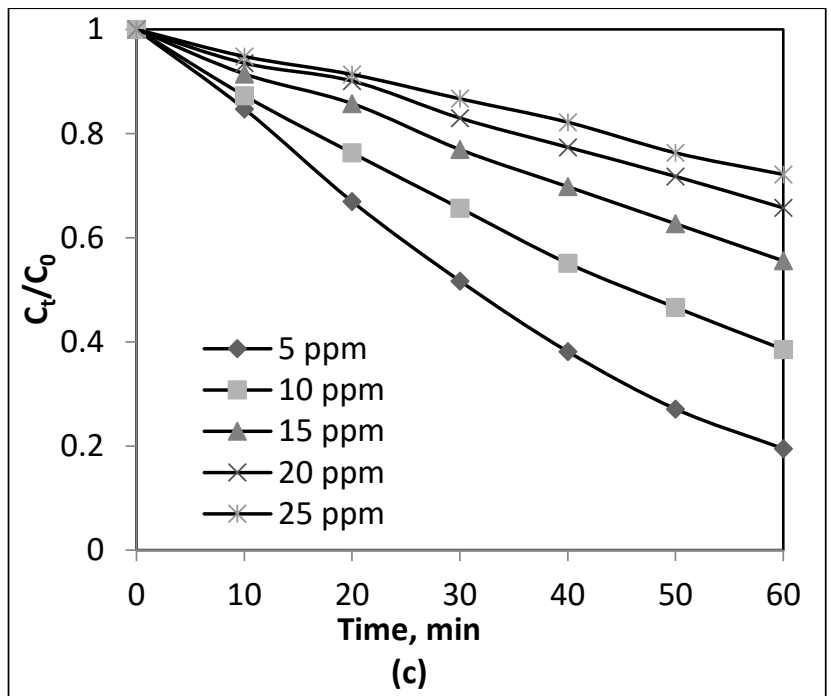

Fig. 2: Effect of (a) sonolysis alone (b) photolysis alone and (c) sonophotolysis on solution concentration [UV-C, Ultrasonic power of 120W].

al., 2011). Under such conditions, the amount of $\cdot \mathrm{OH}$ produced by water sonolysis is sufficient to degrade the RB5 at lower concentration (5) (Mowla et al., 2014). However, an increase in the dye molecules per unit volume leads to an increase in the probability of collision between the dye and hydroxyl radicals. Since the lifespan of hydroxyl radical is extremely short (few nanoseconds), they can only react as soon as they formed (Joseph et al., 2016). Many of the $\cdot \mathrm{OH}$ radicals generated would recombine to form $\mathrm{H}_{2} \mathrm{O}_{2}$ which reduces the efficiency of the sonolytic system (6) (Stock et al., 2000; Ince et al., 2001; Madhavan et al., 2010; Rao et al., 2012). Although the apparent degradation of RB5 is relatively easy at a lower concentration, an effective mineralization of RB5 intermediate byproducts is hard to obtain at a reasonable rate using ultrasound alone (7).

Konstantyinou \& Albanis, (2004) reported that the UV-screening effect of the dye itself which absorbs a part of a light photon decreases the rate of photolysis and sonophotolysis. Therefore, only fewer 
$\cdot \mathrm{OH}$ is available to oxidize the dye molecules in higher concentrations. Sakthivel et al., (2003) mentioned that the rate determining step may be the formation of hydroxyl radical since they have a preference in attacking aromaticringed compound. Therefore, only fewer $\cdot \mathrm{OH}$ is available to oxidize the dye molecules in higher concentrations.

$$
\begin{aligned}
& \mathrm{H}_{2} \mathrm{O} \stackrel{\text { us }}{\longrightarrow} \cdot \mathrm{H}^{+} \cdot{ }^{\circ} \mathrm{H} \\
& \cdot \mathrm{OH}+{ }^{\cdot} \mathrm{OH} \longrightarrow \mathrm{H}_{2} \mathrm{O}_{2} \\
& \mathrm{RB} 5+\cdot{ }^{\circ} \mathrm{OH} \longrightarrow \text { Degradation products }
\end{aligned}
$$

\section{Effect of pH}

Fig. 3 shows RB5 degradation as a function of irradiation time during sonolysis, photolysis and sonophotolysis under acidic, alkaline, and neutral conditions. The experiments were conducted with different initial $\mathrm{pH}$ values i.e., $\mathrm{pH} 3, \mathrm{pH} 7$ and $\mathrm{pH} 10$ using a constant dye concentration (20ppm) for 60 minutes.
The literature suggests that $\mathrm{pH}$ is a major factor influencing the rate of degradation for this target pollutant during the sonophotolytic process. From Fig. 3(a), sonophotolysis of RB5 can be seen to work best under alkaline solution conditions. $67.7 \%$ of RB5 was degraded within the $1 \mathrm{~h}$ experimental period under $\mathrm{pH} 10$ condition. From Fig. 3(b), sonolysis alone only degrade $12-13 \%$ of RB5 at different initial $\mathrm{pH}$ of the solution. Therefore, in this case, the sonolytic degradation of RB5 does not depend on $\mathrm{pH}$ of the solution. Similar findings were found in sonochemical degradation of textile dyes using a similar type of sonicator with the frequency of 35 kHz (Gayathri et al., 2010).

Photolysis in acidic $\mathrm{pH}(\mathrm{pH} 3)$ yielded only $30.3 \%$ of RB5 degradation. Similar findings were reported in the photolytic degradation of real textile wastewater using UV-B indicating that photolysis alone may not be effective (Cardoso et al., 2016). In the order of increasing efficiency, $\mathrm{pH} 7$ was the least favourable condition followed by acidic medium $(\mathrm{pH} 3)$, while $\mathrm{pH} 10$ was

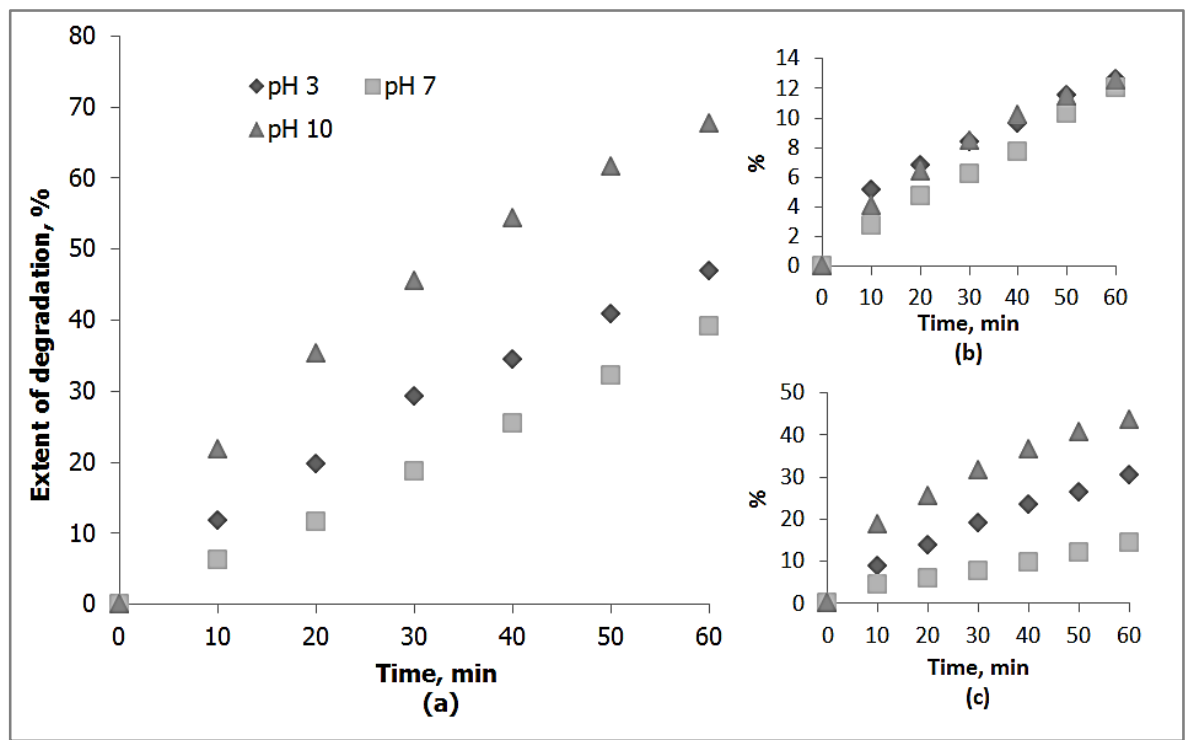

Fig. 3: Effect of $\mathrm{pH}$ a) Sonophotolysis b) Sonolysis and c) Photolysis of RB5. [ $\mathrm{C}_{\mathrm{o}}=20 \mathrm{ppm}$, UVC, Ultrasonic power of $120 \mathrm{~W} 1$ 
most favorable for both RB5 photolytic and sonophotolytic degradation. Similar findings were reported for the photolysis of organic pollutant, bisphenol S, which yielded higher photodegradation rate in alkaline condition (Cao et al., 2012). This is because the presence of more hydroxide ions which are available to oxidize into hydroxyl radical increases both the photolytic and sonophotolytic degradation rate. Additionally, higher $\mathrm{pH}$ also favors the decomposition of $\mathrm{H}_{2} \mathrm{O}_{2}$ into hydroxyl radicals by sonophotolysis (Monteagudo et al., 2014).

\section{Effect of Different Irradiation Wavelength}

The different light irradiation experiments were performed to give a better understanding on the irradiation wavelengths (from shorter to longer) in photolysis and sonophotolysis. Fig. 4 shows the degradation efficiency for sonolysis, photolysis and sonophotolysis separately under different irradiation wavelength. There was no significant change in the RB5 concentration observed throughout the hour for photolysis using UVA, UVB, and solar irradiation. Less than $1 \%$ degradation efficiency was recorded for UV-A and UV-B photolysis. Solar irradiation did not show any significant effect on the degradation rate of RB5 in both photolysis and sonophotolsis. In this study, it is conclusively shown that the UV-C photolysis and sonophotolysis was far superior to UV-A, UV-B or solar radiation related processes in degrading RB5. The inefficiency of UV-A and UV-B and solar photolysis reaction should be attributed to the low photon energy of $365 \mathrm{~nm}, 311 \mathrm{~nm}$, and $610 \mathrm{~nm}$ wavelength respectively, coupled with the lack of sufficient $\cdot \mathrm{OH}$ generation in the reaction system (Table 1 ). Similar findings were reported that UV-C was found far more effective than UV-A in degrading pharmaceuticals, sulfamethoxazole (SMX) and trimethoprim (TMP) in an aqueous solution (Kim et al., 2015).

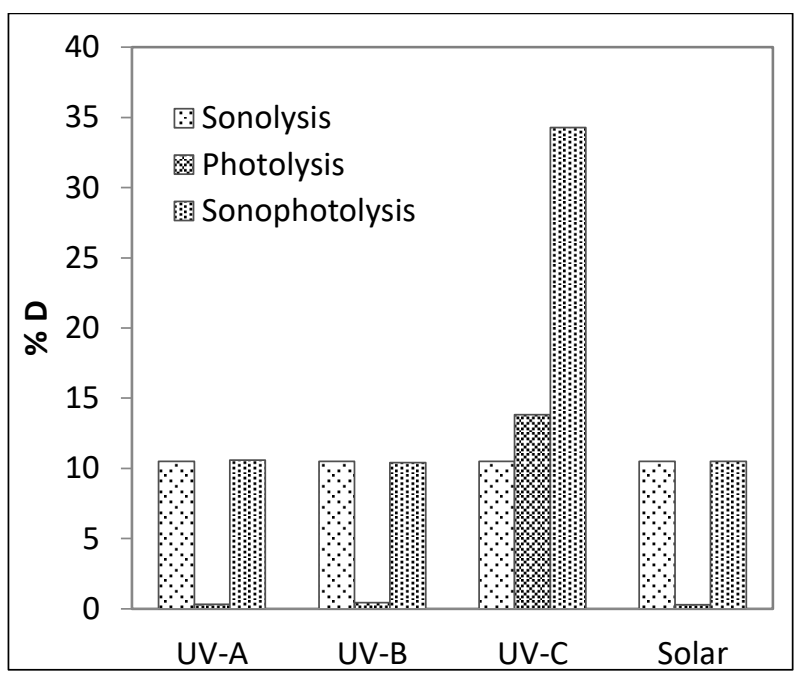

Fig. 4: Effect of different UV irradiation on photolysis and sonophotolysis. $\left[C_{o}=20 p p m\right.$, Ultrasonic power of 120W]

$2 \mathrm{H}_{2} \mathrm{O} \longleftrightarrow \mathrm{H}_{3} \mathrm{O}^{+}+\mathrm{OH}^{-}$

(self ionization of water)

$\mathrm{OH}^{-}+\mathrm{hv}(254 \mathrm{~nm}) \rightarrow \cdot \mathrm{OH}$

The photolytic degradation of RB5 was due to the effect of UV-C radiation of water, which causes sufficient amounts of hydroxyl radicals to be generated (8-9). The lamp photon irradiance was fixed at $10 \mathrm{~mW} / \mathrm{cm}^{2}$ and the total irradiating surface of the lamp was $91.7 \mathrm{~cm}^{2}$, giving the calculated total UV-C radiant flux of $0.917 \mathrm{~W}$. Therefore, the calculated total energy output would be $5.72 \times 10^{18} \mathrm{eV}$ per second. The UV-C lamp has maximum 
wavelength peak of $254 \mathrm{~nm}$. This corresponds to a total energy of $4.88 \mathrm{eV}$.

$$
\begin{aligned}
E & =\frac{6.626 \times 10^{-34} \mathrm{Js} \times 3.0 \times 10^{8} \mathrm{~ms}^{-1}}{254 \times 10^{-9} \mathrm{~m}} \\
& =7.8 \times 10^{-19} \mathrm{~J}
\end{aligned}
$$

Total energy $(\mathrm{eV})$

$=7.8 \times 10^{-19} \times 6.242 \times 10^{18} \mathrm{eV}$

$=4.88 \mathrm{eV}$

The energy required to eject an electron from the oxygen atom in hydroxide ion is $13.6 \mathrm{eV}$, which is the first ionization of oxygen atom. The electron originates from the fourth electron in the $2 p_{x}$ suborbital of the oxygen atom part of the hydroxide ions which then yield hydroxyl radicals. At any given second, $447.50 \mathrm{eV} \mathrm{cm}^{2}$ of the lamp can convert $33 \cdot \mathrm{OH}$ radicals. Total energy output (eV) and a number of $\cdot \mathrm{OH}$ radicals formed with respect to different light sources are tabulated Table 1.

Table 1. Number of $\cdot \mathrm{OH}$ radicals formed with respect to different light sources

\begin{tabular}{lcccc}
\hline $\begin{array}{c}\text { Light } \\
\text { source }\end{array}$ & $\begin{array}{c}\text { Radiant } \\
\text { flux (W) }\end{array}$ & $\begin{array}{c}\text { Total } \\
\text { energy } \\
(\mathbf{e V})\end{array}$ & $\begin{array}{c}\text { Total } \\
\text { energy } \\
\text { output } \\
\left(\mathbf{e V} \mathbf{~ c m}^{2}\right)\end{array}$ & $\begin{array}{c}\text { Number } \\
\text { of } \cdot \mathbf{O H} \\
\text { radicals } \\
\text { formed }\end{array}$ \\
\hline UV-A & 0.917 & 3.40 & 311.78 & 23 \\
UV-B & 0.917 & 3.99 & 365.88 & 27 \\
UV-C & 0.917 & 4.88 & 447.50 & 33 \\
Solar & 0.864 & 2.03 & 175.39 & 14 \\
\hline
\end{tabular}

\section{Degradation Pattern}

The absorbance against time graph in Fig. 5 shows that sonophotolysis using UV$C$ in alkaline condition shows a significant drop in absorbance compared to acidic medium. Fig. 5 depicts the temporal changes in the UV-vis absorbance spectrum of dye samples taken at different exposure times. As shown, it is clearly evident that RB5 exhibits three characteristic absorbance peaks which are progressively reduced upon coming into contact with oxidizing species generated from sonophotolysis. It is also clearly observed (depicted by black dots) that the $\lambda$ max for RB5 as shifted in each spectrum in comparison to the initial value $(595 \mathrm{~nm})$. The peaks appeared to be shifted to the left, signifying hypsochromic (blue) shift (Joseph et al., 2016; Lucas et al., 2006). The peak at $595 \mathrm{~nm}$ in the visible region is a typical characteristic of the chromophore containing long conjugated $\pi$ system, link by two azo double bond group of RB5 (Damodar \& You, 2010).

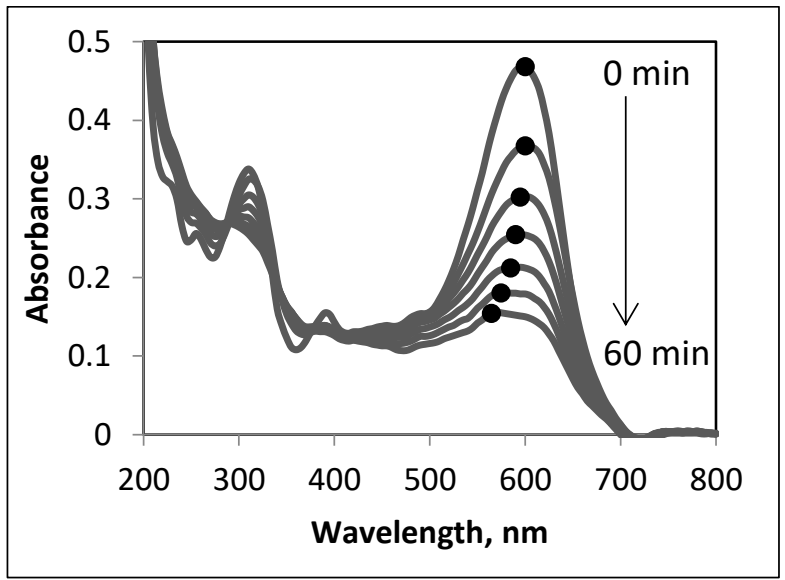

Fig. 5: Degradation pattern of RB5 $\left[C_{0}=20 p p m, p H\right.$ 10, UV-C, Ultrasonic power of $120 \mathrm{~W}]$

Song et al., (2007) documented that the absorption peak at $312 \mathrm{~nm}$ and $254 \mathrm{~nm}$ in UV region are the characteristic peak of naphthalene and benzene ring structure respectively which are assign to $\pi-\pi^{*}$ transition of the electron. The intermediates formed prior to 
photolysis were naphthionic acid, 1-amino-

2-napthol, 2-napthnol, and 1,2napthquinone. The cleavage of the naphthalene and benzene rings would be initiated by the attack of, $\cdot \mathrm{OH}_{1}$ and $\mathrm{O}_{2}{ }^{\cdot-}$ generated in both processes. The degradation of RB5 mainly occurred from the ROS generated in bulk solution rather that at surface catalysts when using $\mathrm{SrTiO}_{3} / \mathrm{CeO}_{2}$ as the catalyst (10-14) (Song et al., 2007). The preferred sites of attack on RB5 (Fig. 6) by reactive oxidizing species were the 1, 4, 9, 15, 21, and 24 positions. The bonds $C(4)-N(7), C(9)-N(8), C(15)$ $\mathrm{N}(19)$, and $\mathrm{C}(21)-\mathrm{N}(20)$ were probably cleaved and led to the degradation of RB5 in the bulk solution.

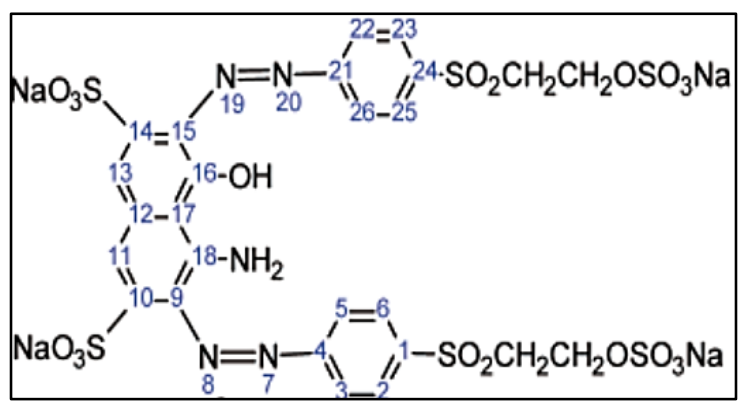

Fig. 6: Molecular structure of RB5

$$
\begin{aligned}
& \mathrm{RB} 5+\mathrm{hv} \rightarrow \mathrm{RB}^{*} \rightarrow \mathrm{X}+\mathrm{Y} \\
& \mathrm{X}+\mathrm{O}_{2} \rightarrow \mathrm{XOO}^{\cdot} \rightarrow \mathrm{X}^{+}+\mathrm{O}_{2}^{\cdot-} \\
& \mathrm{Y}+\mathrm{O}_{2} \rightarrow \mathrm{YOO}^{\cdot} \rightarrow \mathrm{Y}^{+}+\mathrm{O}_{2}^{\cdot-} \\
& \mathrm{RB}^{*}+\mathrm{O}_{2} \rightarrow \mathrm{RB}^{\cdot+}+\mathrm{O}_{2} \\
& \mathrm{RB}^{*}+\mathrm{O}_{2}^{\cdot-} \rightarrow \text { products } \\
& \mathrm{RB}^{\cdot+}+\mathrm{H}_{2} \mathrm{O} \rightarrow \text { products }+\mathrm{OH}^{-}
\end{aligned}
$$

\section{Synergism and Rate Constant}

In this study, the sonolysis, photolysis and sonophotolytic degradation of the followed the first order kinetic reaction where the plots of $\ln \left(C_{0} / C_{t}\right)$ against time. Damodar \& You, (2010) pointed out that usually dye degradation follows first order kinetics. The effect of RB5 solution concentration and initial $\mathrm{pH}$ on synergism between sonolysis and photolysis was calculated using (2). According to this equation if $S>0, S=0$ and $S<0$, the effect is classified as synergistic, additive and antagonistic respectively (Gentili et al., 2009; Joseph et al., 2011). But, there appears to be a synergistic effect between sonolysis and photolysis since rate constants of the combined process are greater than the sum of the rate constants of the individual processes. The beneficial effect of coupling sonolysis and photolysis may be attributed to increased production of hydroxyl radicals in the reaction mixture.

As previously established, $\mathrm{H}_{2} \mathrm{O}_{2}$ is produced during sonication by the interaction of US-generated radical species, either directly $\left(2 \cdot \mathrm{OH} \rightarrow \mathrm{H}_{2} \mathrm{O}_{2}\right.$, $2 \mathrm{HO}_{2} \cdot \mathrm{H}_{2} \mathrm{O}_{2}+\mathrm{O}_{2}$ ) or with water molecules $\left(\mathrm{O} \cdot+\mathrm{H}_{2} \mathrm{O} \rightarrow \mathrm{H}_{2} \mathrm{O}_{2}\right)$. Since the presence of $\mathrm{H}_{2} \mathrm{O}_{2}$ in the sonolysis process was confirmed, the hybrid process of US/UV also incorporates the advanced oxidation process of $U V / \mathrm{H}_{2} \mathrm{O}_{2}$. The influence of UV light intensity on the synergistic effect of the sonophotolytic process likely results from the balance between available $\mathrm{H}_{2} \mathrm{O}_{2}$ and photon intensity ( $\mathrm{Xu}$ et al., 2016). The proposed sonophotolysis 
scheme is described in Fig. 7. Results also showed that the RB5 sonophotodegradation rate followed a first order rate with the kinetic constant increasing with the decrease in solution concentration since more oxidizing species are available at lower solution concentration. From Table $\mathbf{2}$ and $\mathbf{3}$, it is evident that sonophotodegradation resulted in synergistic effect with a higher rate constant value compared to sonolysis and photolysis alone. The synergistic effect resulted from an increase in the $\left({ }^{\circ} \mathrm{OH}\right)$ radicals due to the photolysis of $\mathrm{H}_{2} \mathrm{O}_{2}$ formed by sonolysis process. The highest rate constant for sonophotodegradation of $0.0254 \mathrm{~min}^{-1}$ was quantified when using $5 \mathrm{ppm}$ of RB5 in natural $\mathrm{pH}$ followed by $20 \mathrm{ppm}$ of RB5 at pH10 $\left(0.0194 \mathrm{~min}^{-1}\right)$.

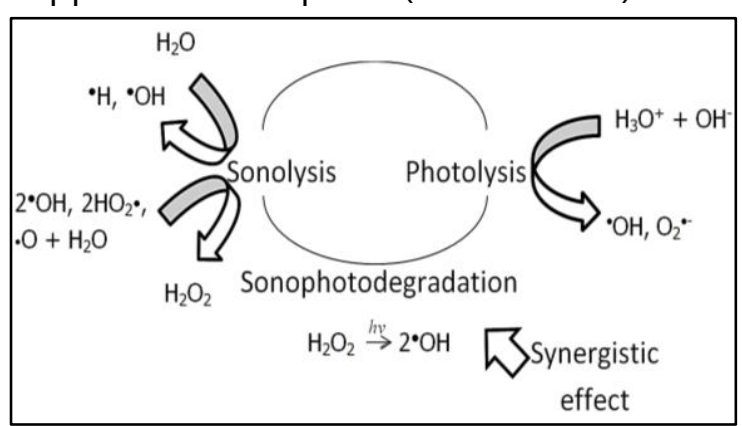

Fig. 7: Degradation scheme of sonophotolysis

\section{Cavitational Yield from Ultrasonic Bath}

The effectiveness of sonochemical reactor for larger scale treatment anionic dye, RB5 containing wastewater degradation has been investigated by carrying out experiments in the available ultrasonic bath with an operating frequency of $35 \mathrm{kHz}$ and rated power output of $120 \mathrm{~W}$ at operating capacity of $0.5 \mathrm{~L}$. The power density (on the basis of rated power input) in the case of the ultrasonic bath was found as $300 \mathrm{~W} / \mathrm{L}$. The sample calculation of cavitational yield obtained for the case of the ultrasonic bath is explained this paper.

Volume and concentration of the treated sample solution: $0.5 \mathrm{~L}$ of the $20 \mathrm{ppm}$ $\mathrm{RB} 5$ solution with reacting period of $60 \mathrm{~min}$.

Electric power supplied: $120 \mathrm{~W}$

$$
\begin{aligned}
& \text { Power density } \begin{aligned}
(\mathrm{J} / \mathrm{L}) & =\frac{120 \times 60 \times 60}{0.5} \\
& =8.64 \times 10^{5} \mathrm{~J} / \mathrm{L}
\end{aligned} \\
& \text { Degaradation obtained } \begin{aligned}
\left(\frac{\mathrm{mg}}{\mathrm{L}}\right) & =\frac{10.49 \times 20}{100} \\
& =2.098 \frac{\mathrm{mg}}{\mathrm{L}}
\end{aligned}
\end{aligned}
$$

Table 2. Synergistic effect of sonolysis, photolysis, and sonophotolysis at different initial $\mathrm{pH}$ of solution

\begin{tabular}{llcccc}
\hline $\mathbf{p H}$ & $\begin{array}{c}\text { Degradation } \\
\text { process }\end{array}$ & $\begin{array}{c}\text { Rate constant } \boldsymbol{k} \\
\left(\mathbf{m i n}^{-1}\right)\end{array}$ & $\mathbf{R}^{\mathbf{2}}$ & $\begin{array}{c}\text { Synergy } \\
(\%)\end{array}$ & $\begin{array}{c}\text { Synergistic } \\
\text { effect }\end{array}$ \\
\hline 3 & Sonolysis & 0.0025 & 0.8740 & & \\
& Photolysis & 0.0064 & 0.9754 & 16.82 & Synergistic \\
& Sonophotolysis & 0.0107 & 0.9959 & & \\
\hline 5.64 & Sonolysis & 0.0020 & 0.9532 & & \\
$($ natural pH) & Photolysis & 0.0025 & 0.9556 & 31.82 & Synergistic \\
& Sonophotolysis & 0.0066 & 0.9890 & & \\
\hline 7 & Sonolysis & 0.0021 & 0.9910 & & \\
& Photolysis & 0.0026 & 0.9709 & 38.96 & Synergistic \\
& Sonophotolysis & 0.0077 & 0.9837 & & \\
\hline 10 & Sonolysis & 0.0025 & 0.9244 & & \\
& Photolysis & 0.0108 & 0.8913 & 31.44 & Synergistic \\
& Sonophotolysis & 0.0194 & 0.9925 & & \\
& & & & &
\end{tabular}


Table 3. Synergistic effect of sonolysis, photolysis, and sonophotolysis at different initial concentration of RB5

\begin{tabular}{llcccc}
\hline Concentration & $\begin{array}{c}\text { Degradation } \\
\text { process }\end{array}$ & $\begin{array}{c}\text { Rate constant } \boldsymbol{k} \\
\left(\mathbf{m i n}^{-1}\right)\end{array}$ & $\mathbf{R}^{\mathbf{2}}$ & $\begin{array}{c}\text { Synergy } \\
\mathbf{( \% )}\end{array}$ & $\begin{array}{c}\text { Synergistic } \\
\text { effect }\end{array}$ \\
\hline $5 \mathrm{ppm}$ & Sonolysis & 0.0052 & 0.9992 & & \\
& Photolysis & 0.0124 & 0.9875 & 30.71 & Synergistic \\
& Sonophotolysis & 0.0254 & 0.9788 & & \\
\hline $10 \mathrm{ppm}$ & Sonolysis & 0.0037 & 0.9739 & & \\
& Photolysis & 0.0059 & 0.9871 & 36.84 & Synergistic \\
& Sonophotolysis & 0.0152 & 0.9937 & & \\
\hline $15 \mathrm{ppm}$ & Sonolysis & 0.0026 & 0.9395 & & \\
& Photolysis & 0.0037 & 0.9796 & 32.25 & Synergistic \\
& Sonophotolysis & 0.0093 & 0.9911 & & \\
& Sonolysis & 0.0020 & 0.9532 & & \\
& Photolysis & 0.0025 & 0.9556 & 31.82 & Synergistic \\
& Sonophotolysis & 0.0066 & 0.9890 & & \\
\hline $25 \mathrm{ppm}$ & Sonolysis & 0.0014 & 0.9826 & & \\
& Photolysis & 0.0017 & 0.9883 & 40.40 & Synergistic \\
& Sonophotolysis & 0.0052 & 0.9903 & & \\
\hline
\end{tabular}

Cavitational yield $(\mathrm{mg} / \mathrm{J})=\frac{2.098}{8.64 \times 10^{5}}$

$$
=2.43 \times 10^{-6} \mathrm{mg} / \mathrm{J}
$$

The net cavitational yield obtained for the ultrasonic bath was $2.43 \times 10^{-6} \mathrm{mg} / \mathrm{J}$. It was found that the cavitational activity is uniform in the case of ultrasonic bath compared to the ultrasonic horn (Barik et al., 2016). Hence it can be emphasized to dissipate same power through larger irradiation areas of transducer bound sonochemical system to get uniform distribution of the cavitational activity.

\section{CONCLUSIONS}

The sonolytic, photolytic and sonophotolytic degradation of RB5 were studied without the presence of any catalyst as the usage of catalyst contributes to additional operational cost in industrial application. The rate of degradation values was calculated for the hybrid system and individual processes. It was concluded from the rate constant values that the combination of sonolysis with photolysis gave a synergistic effect to the degradation process. The result of this study showed that the hybrid US/UV system could effectively degrade the RB5 than the individual processes. Due to the homogeneous nature of reaction system, sonophotolysis does not suffer from limitations of mass transfer; therefore it could be applied for effective degradation of organic pollutant. The results also indicate that the degree of degradation of RB5 was influenced by the RB5 solution concentration and $\mathrm{pH}$. This research is clearly a preliminary approach. The practicality of industrial application would require new trials at a pilot plant scale, but, this study opens another possibility and is relied upon to guide researchers during future work with this method. 


\section{ACKNOWLEDGEMENT}

This research was supported by the Centre of Research \& Innovation of Universiti Putra Malaysia in collaboration with the Centre of Research \& Innovation of Universiti Malaysia Sabah (Grant No. RACE0008-ST-2013) and is gratefully acknowledged.

\section{REFERENCES}

1. Alaton, A. I., Insel, G., Eremektar, G., Babuna, G. F., \& Orhon, D. 2006. Effect of textile auxiliaries on the biodegradation of dyehouse effluent in activated sludge. Chemosphere, 62(9): 1549-1557.

2. Bahena, C. L., Martínez, S. S., Guzmán, D. M., \& Hernández, M. D. R. T. (2008). Sonophotocatalytic degradation of alazine and gesaprim commercial herbicides in $\mathrm{TiO}_{2}$ slurry. Chemosphere, 71(5), 982-989.

3. Barik, A. J., \& Gogate, P. R. (2016). Degradation of 4-chloro 2aminophenol using combined strategies based on ultrasound, photolysis and ozone.Ultrasonics sonochemistry, 28, 90-99.

4. Cao, G., Lu, J., \& Wang, G. (2012). Photolysis kinetics and influencing factors of bisphenol $S$ in aqueous solutions. Journal of Environmental Sciences, 24(5), 846-851.

5. Cardoso, J. C., Bessegato, G. G., \& Zanoni, M. V. B. (2016). Efficiency comparison of ozonation, photolysis, photocatalysis and photoelectrocatalysis methods in real textile wastewater decolorization.Water research, 98, 39-46.

6. Catalkaya, E. C., \& Kargi, F. 2009. Advanced oxidation and mineralization of simazine using Fenton's reagent. Journal of hazardous materials, 168(2): 688-694.

7. Chakma, S., \& Moholkar, V. S. (2016). Mechanistic analysis of sono-photolysis degradation of carmoisine. Journal of Industrial and Engineering Chemistry, 33, 276-287.

8. Damodar, R. A., \& You, S. J. 2010. Performance of an integrated membrane photocatalyticreactor for the removal of Reactive Black 5 . Separation and Purification Technology, 71(1): 44-49.

9. Durán, A., Monteagudo, J. M., Sanmartín, I., \& García-Díaz, A. (2013). Sonophotocatalytic mineralization of antipyrine in aqueous solution. Applied Catalysis B: Environmental, 138, 318325.

10. Gayathri, P., Dorathi, R. P. J., \& Palanivelu, K. (2010). Sonochemical degradation of textile dyes in aqueous solution using sulphate radicals activated by immobilized cobalt ions. Ultrasonics sonochemistry, 17(3), 566-571.

11. Gentili, P. L., Penconi, M., Ortica, F., Cotana, F., Rossi, F., \& Elisei, F. (2009). Synergistic effects in hydrogen production through water sonophotolysis catalyzed by new La $2 x$ Ga $2 y$ In 2 (1- x- y) 03 solid solutions. International journal of hydrogen energy, 34(22), 9042-9049.

12. Ince, N. H., \& Tezcanlí, G. (2001). Reactive dyestuff degradation by combined sonolysis and 
ozonation. Dyes and pigments, 49(3), 145-153.

13. Joseph, C. G., Li Puma, G., Bono, A., Taufiq-Yap, Y. H., \& Krishnaiah, D. (2015). Sonolysis, Photolysis, and Sequential Sonophotolysis for the Degradation of 2, 4, 6-Trichlorophenol: The Effect of Solution Concentration. Chemical Engineering Communications, 202(8), 1061-1068.

14. Joseph, C. G., Puma, G. L., Bono, A., Taufiq-Yap, Y. H., \& Krishnaiah, D. (2011). Operating parameters and synergistic effects of combining ultrasound and ultraviolet irradiation in the degradation of $2,4,6$ trichlorophenol.Desalination, 276(1), 303-309.

15. Joseph, C. G., Taufiq-Yap, Y. H., Li Puma, G., Sanmugam, K., \& Quek, K. S. (2016). Photocatalytic degradation of cationic dye simulated wastewater using four radiation sources, UVA, UVB, UVC and solar lamp of identical power output. Desalination and Water Treatment, 57(17), 7976-7987.

16. Konstantyinou, I. K., \& Albanis, T. A. 2004. $\mathrm{TiO}_{2}$-assisted photocatalytic degradation of azo dyes in aqueous solution: kinetic and mechanistic investigations. A review, App.

Catalyst B: Environmental, 49: 1-14.

17. Lucas, M. S., \& Peres, J. A. (2006). Decolorization of the azo dye Reactive Black 5 by Fenton and photo-Fenton oxidation. Dyes and Pigments, 71(3), 236-244.

18. Madhavan, J., Grieser, F., \& Ashokkumar, M. (2010). Degradation of orange- $G$ by advanced oxidation processes. Ultrasonics sonochemistry, 17(2), 338-343.

19. Mezohegyi, G., van der Zee, F. P., Font, J., Fortuny, A., \& Fabregat, A. 2012. Towards advanced aqueous dye removal processes: A short review on the versatile role of activated carbon. Journal of environmental management, 102: 148-164.

20. Mohan, N., \& Balasubramanian, N. 2006. In situ electrocatalytic oxidation of acid violet 12 dye effluent. Journal of hazardous materials, 136(2): 239-243.

21. Monteagudo, J. M., Duran, A., \& San Martín, I. (2014). Mineralization of wastewater from the pharmaceutical industry containing chloride ions by UV photolysis of $\mathrm{H} 2 \mathrm{O} 2 / \mathrm{Fe}$ (II) and ultrasonic irradiation. Journal of environmental management, 141, 6169.

22. Mowla, A., Mehrvar, M., \& Dhib, R. (2014). Combination of sonophotolysis and aerobic activated sludge processes for treatment of synthetic pharmaceutical wastewater. Chemical Engineering Journal, 255, 411-423.

23. Ramírez, C., Saldaña, A., Hernández, B., Acero, R., Guerra, R., Garcia-Segura, S. \& Peralta-Hernández, J. M. 2012. Electrochemical oxidation of methyl orange azo dye at pilot flow plant using BDD technology. Journal of Industrial and Engineering Chemistry.

24. Rao, Y., Yang, H., Xue, D., Guo, Y., Qi, F., \& Ma, J. (2016). Sonolytic and sonophotolytic degradation of Carbamazepine: Kinetic and mechanisms.Ultrasonics sonochemistry, 32, 371-379.

25. Robinson, T., McMullan, G., Marchant, R., \& Nigam, P. 2001. Remediation of 
dyes in textile effluent: a critical review on current treatment technologies with a proposed alternative. Bioresource technology, 77(3): 247-255.

26. Rodríguez, V. M., Hernández-Ramírez, A., Peralta-Hernández, J. M., Bandala, E. R., \& Quiroz-Alfaro, M. A. 2009. Enhancing the electrochemical oxidation of acid-yellow 36 azo dye using boron-doped diamond electrodes by addition of ferrous ion. Journal of hazardous materials, 167(1): 1226-1230.

27. Sakthivel, S., Neppolian, B., Shankar, M. V., Arabindoo, B., Palanichamy, M., \& Murugesan, V. (2003). Solar photocatalytic degradation of azo dye: comparison of photocatalytic efficiency of $\mathrm{ZnO}$ and $\mathrm{TiO}_{2}$. Solar Energy Materials and Solar Cells, 77(1), 65-82.

28. Song, S., Xu, L., He, Z., Chen, J., Xiao, X., \& Yan, B. 2007. Mechanism of the photocatalytic degradation of $\mathrm{Cl}$ Reactive Black 5 at $\mathrm{pH} 12.0$ using $\mathrm{SrTiO}_{3} / \mathrm{CeO}_{2}$ as the catalyst. Environmental science \& technology, 41(16): 5846-5853.

29. Stock, N. L., Peller, J., Vinodgopal, K., \& Kamat, P. V. (2000). Combinative sonolysis and photocatalysis for textile dye degradation. Environmental science \& technology, 34(9), 1747-1750.

30. Wang, C., Yediler, A., Lienert, D., Wang, Z., \& Kettrup, A. 2003. Ozonation of an azo dye C.I Remazol Black 5 and toxicological assessment of its oxidation

products. Chemosphere, 52(7): 12251232.

31. Xu, L. J., Chu, W., \& Graham, N. (2013). Sonophotolytic degradation of dimethyl phthalate without catalyst: analysis of the synergistic effect and modeling. Water research, 47(6), 19962004.

32. Xu, L. J., Chu, W., Lee, P. H., \& Wang, J. (2016). The mechanism study of efficient degradation of hydrophobic nonylphenol in solution by a chemicalfree technology of sonophotolysis. Journal of hazardous materials, 308, 386-393.

33. Zhou, T., Lu, X., Wang, J., Wong, F. S., \& Li, Y. (2009). Rapid decolorization and mineralization of simulated textile wastewater in a heterogeneous Fenton like system with/without external energy. Journal of Hazardous Materials, 165(1), 193-199. 